OPEN ACCESS

Edited by: James Bernard Konopka, Stony Brook University, United States

Reviewed by:

Peter William Piper, University of Sheffield,

United Kingdom

Andrew Truman, University of North Carolina at

Charlotte, United States

Frederic Lamoth

Centre Hospitalier Universitaire

Vaudois (CHUV), Switzerland

${ }^{*}$ Correspondence: Shujuan Sun

sunshujuan888@163.com

Received: 28 September 2017 Accepted: 07 December 2017 Published: 19 December 2017

Citation:

Gong Y, Li T, Yu C and Sun S (2017)

Candida albicans Heat Shock Proteins and Hsps-Associated Signaling Pathways as Potential Antifungal

Targets.

Front. Cell. Infect. Microbiol. 7:520. doi: 10.3389/fcimb.2017.00520

\section{Candida albicans Heat Shock Proteins and Hsps-Associated Signaling Pathways as Potential Antifungal Targets}

\author{
Ying Gong ${ }^{1}$, Tao $\mathrm{Li}^{2}$, Cuixiang $\mathrm{Yu}^{3}$ and Shujuan Sun ${ }^{4 *}$ \\ ${ }^{1}$ School of Pharmaceutical Sciences, Shandong University, Jinan, China, ${ }^{2}$ Intensive Care Unit, Qianfoshan Hospital Affiliated \\ to Shandong University, Jinan, China, ${ }^{3}$ Respiration Medicine, Qianfoshan Hospital Affiliated to Shandong University, Jinan, \\ China, ${ }^{4}$ Department of Pharmacy, Qianfoshan Hospital Affiliated to Shandong University, Jinan, China
}

In recent decades, the incidence of invasive fungal infections has increased notably. Candida albicans (C. albicans), a common opportunistic fungal pathogen that dwells on human mucosal surfaces, can cause fungal infections, especially in immunocompromised and high-risk surgical patients. In addition, the wide use of antifungal agents has likely contributed to resistance of $C$. albicans to traditional antifungal drugs, increasing the difficulty of treatment. Thus, it is urgent to identify novel antifungal drugs to cope with C. albicans infections. Heat shock proteins (Hsps) exist in most organisms and are expressed in response to thermal stress. In C. albicans, Hsps control basic physiological activities or virulence via interaction with a variety of diverse regulators of cellular signaling pathways. Moreover, it has been demonstrated that Hsps confer drug resistance to C. albicans. Many studies have shown that disrupting the normal functions of $C$. albicans Hsps inhibits fungal growth or reverses the tolerance of C. albicans to traditional antifungal drugs. Here, we review known functions of the diverse Hsp family, Hsp-associated intracellular signaling pathways and potential antifungal targets based on these pathways in $C$. albicans. We hope this review will aid in revealing potential new roles of $C$. albicans Hsps in addition to canonical heat stress adaptions and provide more insight into identifying potential novel antifungal targets.

Keywords: heat shock proteins, Candida albicans, signaling pathways, antifungal targets, virulence

\section{INTRODUCTION}

C. albicans causes superficial and potentially life-threatening systemic infections, especially in immunocompromised and high-risk surgical patients (Dimopoulos et al., 2007). Traditional antifungal drugs, such as azoles, polyenes, and echinocandins, mostly target C. albicans' cell envelopes, e.g., ergosterol on the plasma membrane or glucan on the cell wall (Odds et al., 2003). These antifungal drugs are extensively used in the clinic because of their high efficacy. However, traditional antifungal drugs have become increasingly ineffective against $C$. albicans infections due to multiple factors and resistance of C. albicans to these drugs has emerged more frequently (Kriengkauykiat et al., 2011; Wirk, 2011). Thus, it is urgent to develop novel antifungal agents based on identified potential targets in C. albicans (Wirk, 2011; Cuenca-Estrella, 2014). 
The Hsp family was first identified in Drosophila melanogaster in response to thermal stress (Tissières et al., 1974; Brown et al., 2014). Subsequently, it was shown that Hsps are evolutionarily conserved in most organisms and are activated by additional, non-thermal stressors, e.g., heavy metals and oxidative stress (Burnie et al., 2006; Soo et al., 2008; Wirk, 2011; CuéllarCruz et al., 2014). Furthermore, many studies have revealed important roles for Hsps in the growth and virulence of $C$. albicans (Leach et al., 2012b; Becherelli et al., 2013; O'meara and Cowen, 2014). Hsps are widely distributed in C. albicans and involved in many cellular pathways, such as calcium-calcineurin, MAPK, Ras1-cAMP-PKA, and cell cycle control signaling. Many signaling molecules in these pathways are client proteins of Hsps. Moreover, many studies have demonstrated that Hsps confer C. albicans resistance to antifungal drugs by regulating these signaling pathways. Therefore, targeting Hsps pharmacologically or genetically could enhance the sensitivity of C. albicans to traditional antifungal drugs and reduce its pathogenicity (Fiori et al., 2012; Mayer et al., 2013; Li and Sun, 2016). Thus, Hsps and other signaling molecules of Hsps-associated pathways are potential novel antifungal targets against candidiasis. Hspassociated signaling pathways and potential antifungal targets based on these signaling pathways in C. albicans are illustrated in Figure 1. The research identifying antifungal agents that target Hsps and Hsp-associated signaling pathways is summarized in Table 1.

In this article, we will review the functions of Hsps in $C$. albicans, the roles of Hsps in various intracellular signaling pathways and potential antifungal targets based on Hspassociated signaling pathways. Several elements of the Hsp family may represent novel antifungal targets against $C$. albicans infections.

\section{FUNCTIONS OF HSPS IN C. ALBICANS}

As a class of molecular chaperones, Hsps have multiple broad functions within organisms. Generally, Hsps are classified according to their molecular size (Powers and Workman, 2007). In C. albicans, six kinds of Hsps with varying molecular sizes have been identified. Four of them, Hsp104, Hsp90, Hsp70, and Hsp60, are adenosine triphosphate (ATP)-dependent high molecular mass Hsps. The other two, Hsp12 and Hsp21, are ATPindependent low molecular mass Hsps with sizes ranging from 12 to $42 \mathrm{kDa}$ (Jaya et al., 2009). Most of these Hsps play significant roles in the growth and virulence of $C$. albicans.

\section{Hsp104}

As a kind of heat-induced molecular disaggregase, Hsp104 was first discovered in Saccharomyces cerevisiae (S. cerevisiae) (Sanchez and Lindquist, 1990; Glover and Lindquist, 1998; Jaya et al., 2009). In C. albicans, Hsp104 expression increases after transient exposure of cells to high temperature. Hsp104 is a pro-survival mediator in response to increasing temperature, suggesting an important role for this protein in thermotolerance (Sanchez and Lindquist, 1990). In addition, hyphae formed in biofilms by wild-type and Hsp104-reconstituted strains grow in an intertwined appearance. In contrast, hyphae formed by hsp $104 \Delta / \Delta$ mutants show structural defects, appearing patchy and loose. These mutants also show attenuated pathogenicity in Caenorhabditis elegans infection models. These results demonstrate that Hsp104 is required for efficient biofilm formation and contributes to the virulence of C. albicans (Fiori et al., 2012). Hsp104 represents a prospective antifungal target against C. albicans because of the absence of a cytosolic Hsp104 equivalent in human.

\section{Hsp90}

Hsp90 augments virulence factors and confers antifungal drug resistance to common pathogenic fungi, C. albicans, Aspergillus fumigatus, and Cryptococcus neoformans (Cordeiro Rde et al., 2016; Lamoth et al., 2016; Chatterjee and Tatu, 2017). The functions of Hsp90 are modulated by posttranscriptional modifications, mainly including phosphorylation, S-nitrosylation, and acetylation in yeast. Soroka et al. determined 10 major phosphorylation sites regulated by the dedicated phosphatase Ppt1 in the middle or the C-terminal domain of yeast Hsp90. Phosphorylation allows conformational switching and facilitates communication of remote regions within Hsp90 (Soroka et al., 2012). Besides, a cysteine residue located in the C-terminal domains is proved to be conserved in yeast and human Hsp90 family. S-nitrosylation of this residue regulates the functions of Hsp90 fast and efficiently (Martínez-Ruiz et al., 2005; Retzlaff et al., 2009). Moreover, the process of histone acetylation regulated by histone acetyltransferases (HATs) and histone deacetylases (HDACs), also known as lysine deacetylases (KDACs), plays a causative role in regulating gene expression (Trojer et al., 2003). Recent studies showed that Hsp90 acetylation has a profound impact on Hsp90 function. $\mathrm{Li}$ et al. verified that the key acetylation sites on C. albicans Hsp90 are lysine 30 and 271 and substitutions at these residues phenocopy inhibition of Hsp90 (Li et al., 2017). Furthermore, HDAC inhibitors, such as trichostatin A (TSA) and MGCD290, a Hos2 HDAC inhibitor, have been shown to abrogate Hsp90dependent azole resistance in C. albicans. These results illustrate that acetylation regulates Hsp90 function and then governs antifungal drug resistance with broad therapeutic prospects (Smith and Edlind, 2002; Pfaller et al., 2009; Robbins et al., 2012). The challenge of successfully targeting HDACs in the treatment of candidiasis is to develop stable and specific inhibitors capable of distinguishing pathogens from host.

Hsp90 is one of the most intensely studied Hsps in C. albicans. Many studies demonstrate that Hsp90 plays an important role in thermal stability, morphogensis, cell cycle regulation, apoptosis, and drug resistance in C. albicans (Leach et al., 2012b; O'meara and Cowen, 2014). During evolution, C. albicans retained the conserved protein Hsp90, enabling C. albicans to adapt to thermal stress upon colonization of warm-blooded animals or thermally buffered niches (Leach et al., 2012a). Hsp90 governs cellular circuitry required for crucial morphogenetic transitions from yeast to filament (Shapiro et al., 2009, 2012b). Genetically or pharmacologically inhibiting the functions of Hsp90 blocks the growth, maturation, and dispersal of C. albicans biofilms in vitro (Robbins et al., 2011). In addition, the filaments generated by compromised Hsp90 are similar to those seen during cell 


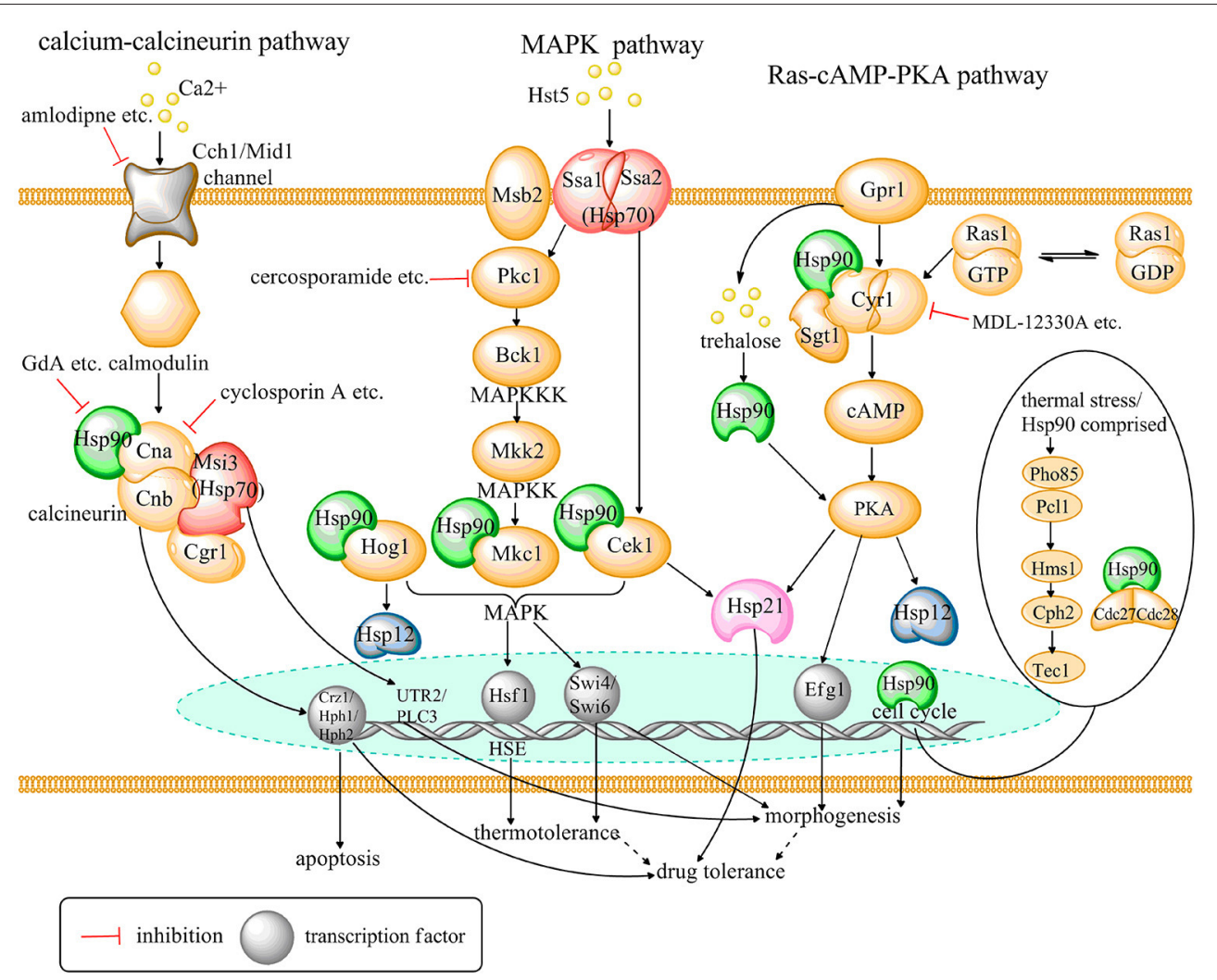

FIGURE 1 | A schematic diagram depicting Hsp-associated signaling pathways and potential antifungal targets based on these pathways in C. albicans. Solid and dashed arrow lines indicate known pathways and putative pathways, respectively, which are predicted in the present study.

cycle arrest. Further studies illustrate that C. albicans Hsp90 regulates the progression of yeast-form growth via interaction with diverse control elements of the cell cycle (Berman, 2006; Senn et al., 2012). In addition, Léger et al. demonstrated that in C. albicans the metacaspase Mcalp appears to degrade several major Hsps, including Hsp90, which weakens cellular defenses and leads to apoptosis (Léger et al., 2015). Hence, Hsp90 is tightly related by apoptosis, and inhibiting $\mathrm{Hsp} 90$ reduces apoptosis in C. albicans (Dai et al., 2012). Furthermore, Hsp90 contributes to the appearance and maintenance of antifungal drug resistance in C. albicans. Impairing the function of Hsp90 in vitro could enhance the efficacy of azoles against $C$. albicans planktonic cells and biofilms (Cowen and Lindquist, 2005; Cowen et al., 2009; Shapiro et al., 2009; Robbins et al., 2011). Hsp90 inhibitors have been shown to have synergistic effects in combination with fluconazole (FLC) against FLC-resistant C. albicans (Cowen et al., 2009; Li et al., 2015), so it follows that interfering with the physiological activity of Hsp90 could be a promising strategy to treat candidiasis (Veri and Cowen, 2014).

The currently available agents that pharmacologically target Hsp90 are primarily Hsp90 inhibitors and anti-Hsp90 antibodies. As mentioned above, high-molecular-mass Hsps are ATP-dependent and the N-terminal domain of Hsp90 is an ATP binding site. This domain is highly conserved across species and is essential for the function of Hsp90 (Jackson,
2013). Currently available Hsp90 inhibitors include radicicol (RAD), geldanamycin (GdA), analogs of GdA, including 17-allylamino-17-dimethoxygeldanamycin(17-AAG) and 17-dimethylaminoethylamino-17-demethoxygeldanamycin(17DMAG) and non-GdA Hsp90 inhibitors, e.g., NVP-HSP990 (HSP990). Each of these binds with the same site in the Nterminus of Hsp90 (Singh et al., 2009; Wirk, 2011; Li et al., 2015). Singh et al. reported that both RAD and GdA reduce the tolerance of $C$. albicans to echinocandins and that each of them exerts synergistic antifungal effects in combination with echinocandins (Singh et al., 2009). Additionally, Cowen et al. found that GdA analogs have synergistic effects in combination with FLC against C. albicans (Cowen et al., 2009). However, GdA and its derivatives have not been fully developed for clinical use because of their limited physiochemical properties and severe renal and gastrointestinal cytotoxicity (Kim et al., 2009). This makes non-GdA Hsp90 inhibitors more attractive for therapeutic development. Li et al. demonstrated that HSP990, a non-GdA Hsp90 inhibitor, synergistically combines with FLC against FLC-resistant C. albicans and C. albicans biofilms both in vitro and in vivo. HSP990 was also shown to exhibit low cytotoxicity in human umbilical vein endothelial cells (Li et al., 2015). This finding is a promising prospect for the clinical application of non-GdA Hsp90 inhibitors to treat C. albicans infections. Although the highly conserved structure of eukaryotic Hsp90 
has hindered the development of Hsp90 inhibitors for clinical application, it is promising to find drugs with low cytotoxicity, like HSP990, and there is future potential to find specific targets of C. albicans Hsp90.

Another way to inhibit Hsp90 is via stimulation of the host immune response (Bugli et al., 2013). Efungumab (Mycograb), a well-known recombinant monoclonal anti-Hsp90 antibody, binds to the middle domain of Hsp90, effectively inhibiting communication between the terminal regions of Hsp90 and preventing necessary conformational changes (Karwa and Wargo, 2009). The discovery of efungumab against C. albicans stemmed from the observation that the presence of anti-Hsp90 antibody is associated with recovery in patients with invasive candidiasis treated with amphotericin B (AMB) (Matthews et al., 1984, 1987). Subsequently, Matthews et al. identified that efungumab exerts antifungal effects alone and synergistically when combined with FLC, AMB, and caspofungin against $C$. albicans in vitro and in vivo (Matthews et al., 2003; Hodgetts et al., 2008). Meanwhile, the in-vivo pharmacodynamics of efungumab have been confirmed in a murine model. After intravenous administration, it is rapidly distributed into tissues and cleared from the circulation (Matthews et al., 2003; Louie et al., 2011). A blinded and randomized clinical trial further explained the effects of efungumab. The use of efungumab plus lipid-associated AMB produces significant clinical improvement compared to the use of amphotericin B plus placebo for patients with invasive candidiasis (Pachl et al., 2006). In consideration of quality concerns, such as heterogeneity in molecular weight and conformational structure, and safety concerns about adverse effects, such as cytokine release syndrome and hypertension, efungumab was not accredited by the Committee for Medicinal Products for Human Use (CHMP) (Bugli et al., 2013). In response to circumvent these problems for more stability, Louie et al. designed a new compound Mycograb C28Y and identified its synergistic effect in combination with AMB against C. albicans in vitro. It is worth noting that Mycograb C28Y lacks efficacy in a murine model with invasive candidiasis (Louie et al., 2011). Besides, the synergistic effect of Mycograb C28Y combined with $\mathrm{AMB}$ against $C$. albicans can be reproduced by a wide range of unrelated proteins, indicating that Mycograb C28Y has multiple targets in C. albicans (Richie et al., 2012). These findings suggest that complex mechanisms of Mycograb C28Y acting on $C$. albicans need to be further explored. Development of anti-Hsp90 antibodies as novel antifungal agents is a promising therapeutic approach for candidiasis in view of their excellent antifungal effects and clinical efficacy. Certainly, animal experiments and clinical trials are required to confirm the safety and efficacy before anti-Hsp90 antibodies could be contemplated for a clinical use in the future.

\section{Hsp70}

Hsp70 is highly conserved among most species from bacteria to mammals. Hsp70 from different sources has similar biochemical properties because of a high degree of conservation of $\mathrm{N}$-terminal domains; each has a high-affinity ATP-binding site and a peptidebinding site (Craig et al., 1993). Cell surface Ssa1 and Ssa2 are the major members of the Hsp70 family in C. albicans (López-Ribot et al., 1996; Eroles et al., 1997). It has been suggested that Ssa1 and Ssa2 exert both positive and negative effects on the growth and virulence of C. albicans. On the one hand, Ssa1 and Ssa2 induce host cell endocytosis leading to C. albicans mediated pathogenic host cell interactions and increased virulence (Sun et al., 2010). C. albicans $\operatorname{Ss} a / \Delta$ mutants exhibit attenuated virulence both in vitro and in vivo. On the other hand, Ssa1 and Ssa2 are receptors of some antimicrobial peptides that exert antifungal effects. For instance, salivary histatin5 (Hst5) has a high affinity for Ssa proteins on the cell wall of $C$. albicans, facilitating the import of Hst5 (Li et al., 2006; Vylkova et al., 2006, 2007), which is required for Hst5 to exert fungicidal activity against $C$. albicans. Moreover, Maneu et al. discovered that C. albicans Ssb1 encoded by SSB1 has $85 \%$ amino acid identity as Ssb1 and Ssb2 of S. cerevisiae Hsp70 family (Maneu et al., 1997). Expression of C. albicans SSB1 complements S. cerevisiae SSB1 SSB2 double mutant phenotype, indicating that $C$. albicans Ssb1 probably act as a molecular chaperone on the translating ribosomes (Maneu et al., 2000). Msi3, while technically in the Hsp70 family is the homolog of the S. cerevisiae Sse1 (human Hsp110). They are regarded as co-chaperones since they are nucleotide exchange factors for Ssa. Msi3 is essential for survival in vitro as well as for the establishment of $C$. albicans infections in a mouse model (Nagao et al., 2012). In addition, the mutant strain tetMsi3, whose expression of MSI is repressed, exhibits higher susceptibility to FLC than does the control strain. Furthermore, tetMsi3 altered the response to FLC from fungistatic activity to fungicidal activity (Cho et al., 2003; Nagao et al., 2012). Thus, compromising Hsp70 inhibits host cell phagocytosis of C. albicans and decreases C. albicans antifungal drug resistance. We believe that Hsp70 would also be a potential antifungal target against candidiasis if it conquers the obstacles of safety caused by highly conserved structure of Hsp70 among eukaryotes.

\section{Hsp60}

As immunodominant antigens in humoral and cellular responses, fungal Hsp60 facilitates powerful immunological reactions (Habich et al., 2006). Cross-reactivity between fungal and human Hsp60 may illustrate a potential link between infection and autoimmunity (47). Expression of C. albicans Hsp60 mRNA increase upon elevated incubation temperature beyond $35^{\circ} \mathrm{C}$ (Raggam et al., 2011). Many studies showed that Hsp60 acts as an immunogenic trigger in orchestrating $C$. albicans-related diseases under thermal stress. These findings may contribute to a deeper understanding of host-pathogen relationships (Rajaiah and Moudgil, 2009; Raggam et al., 2011).

\section{Small Hsps}

Unlike the highly conserved sequences among species of highmolecular-mass Hsps, small Hsps share only a short fragment called the acystall within a conserved sequence of the C-terminus. C. albicans Hsp12 is a small Hsp primarily expressed under different types of stress, like osmotic and thermal stress (Enjalbert et al., 2003; Smith et al., 2004). Enhanced expression of Hsp12 significantly promotes cell adhesion and germination of $C$. albicans, while decreasing the susceptibility of C. albicans to the quorum sensing molecule, farnesol (Davis-Hanna et al., 2008; 


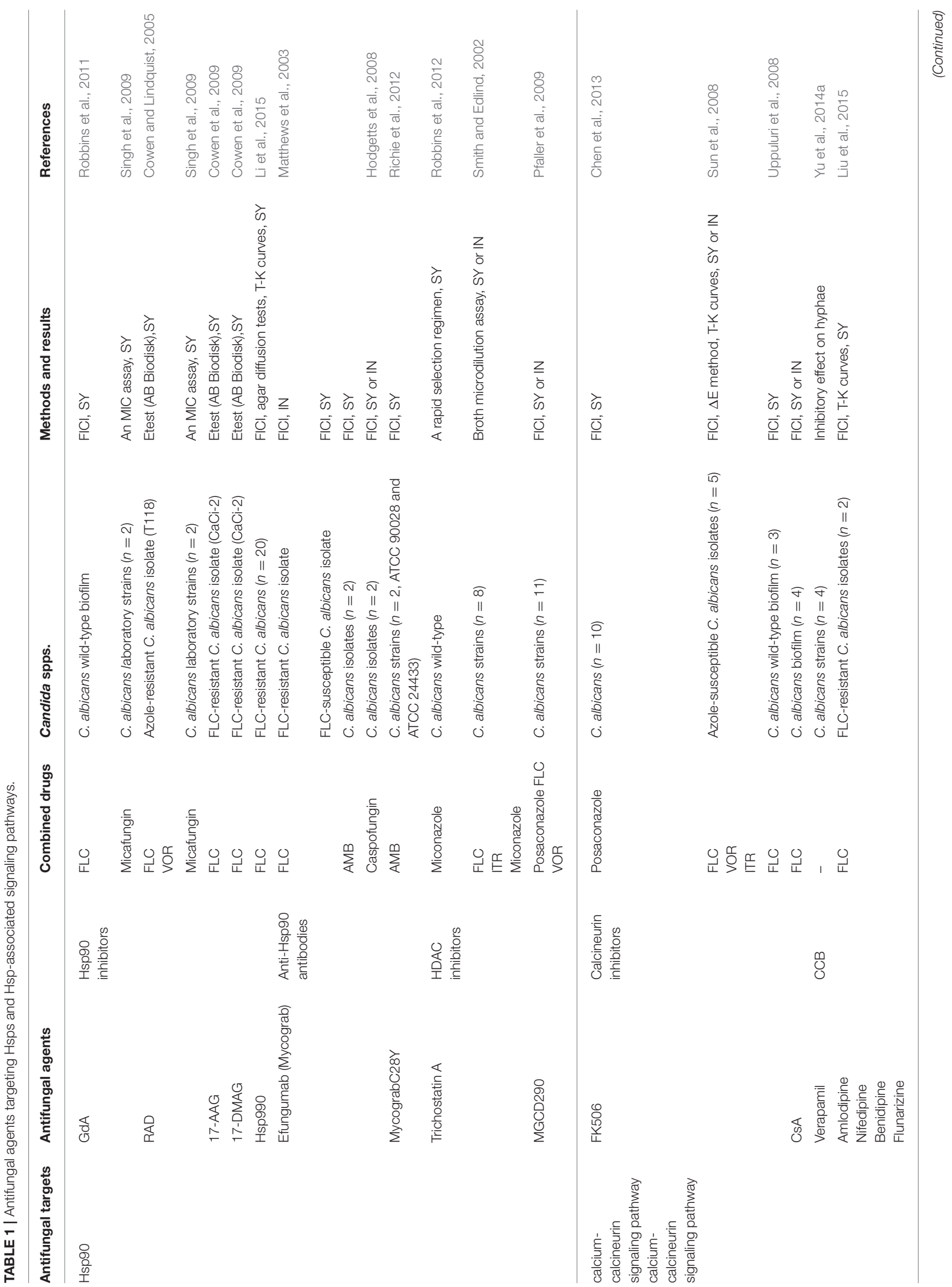


Fu et al., 2012). In addition, C. albicans strains overexpressing HSP12 are sensitive to itraconazole, ketoconazole, and FLC (Fu et al., 2012). Thus, targeted up regulation of Hsp12 expression is a potential antifungal treatment against $C$. albicans.

Hsp21 is another small Hsp crucial for C. albicans to resist specific stressors, including thermal and oxidative stress. Hsp21 is also involved in regulation of glycerol, glycogen, and trehalose homeostasis in response to elevated temperature (Mayer et al., 2012). Additionally, Hsp21 was found to promote the virulence of C. albicans. Hsp21 $\Delta / \Delta$ mutants form significantly shorter hyphae and exhibit defects in invasive growth (Mayer et al., 2012). Furthermore, $h s p 21 \Delta / \Delta$ C. albicans mutants are highly susceptive to a broad range of antifungal drugs (Mayer et al., 2013). Therefore, targeting Hsp21 is a possible treatment strategy for C. albicans infection.

\section{POTENTIAL ANTIFUNGAL TARGETS WITHIN HSPS-ASSOCIATED INTRACELLULAR SIGNALING PATHWAYS IN C. ALBICANS}

Hsps are involved in a wide variety of intracellular signaling pathways in C. albicans. In response to activation of diverse signaling pathways, the heat shock transcription factor (Hsfl) is phosphorylated, resulting in the induction of target HSP gene expression via the heat shock element (HSE) (Nicholls et al., 2009). Thus, therapeutic targeting of Hsps could exert antifungal effects or reverse drug tolerance of C. albicans to antifungal drugs by disrupting Hsp-related signaling pathways.

The number of available Hsp inhibitors is limited; however, drugs targeting other elements of Hsp-related signaling pathways have been shown to exert antifungal effects. In this section, we will review the Hsp-associated signaling pathways in C. albicans and summarize potential antifungal targets in these pathways.

\section{Calcium-Calcineurin Signaling Pathway}

Calcineurin is a conserved calmodulin-dependent phosphatase in pathogenic fungi. Calcineurin is activated by the second messenger calcium and regulates stress responses in fungi (Kraus and Heitman, 2003). Calcineurin is necessary for C. albicans to survive during cell membrane stress, cation stress, alkaline $\mathrm{pH}$, and endoplasmic reticulum stress (Cruz et al., 2002; Steinbach et al., 2007). Elements of the calcium-calcineurin signaling pathway, such as various channels, transporters and other proteins or enzymes, are intimately connected to various physiological processes in C. albicans (Liu et al., 2015; Li and Sun, 2016).

Singh et al. revealed that impairing the calcineurin function phenocopies inhibition of Hsp90 (Singh et al., 2009), both of which result in decreased drug resistance of $C$. albicans to azoles. Calcineurin is a heterodimer composed of a catalytic subunit (either Cna1 or Cna2) and an activating regulatory subunit (Cnb1) (Cowen and Lindquist, 2005). It is widely accepted that calcineurin is a client protein of Hsp90, which binds to the catalytic subunit of calcineurin in C. albicans (Cowen, 2009; Li and Sun, 2016). In addition, downstream targets of 
calcineurin include the transcription factors Hph1, Hph2, and Crz1, which were initially discovered in S. cerevisiae as targets related to stress adaption and drug resistance (Heath et al., 2004; Karababa et al., 2006). Both Crz1 and Hph1 modulate Hsp90dependent drug resistance (Cowen et al., 2006), and the key mediator of Hsp90-dependent drug resistance is thought to be calcineurin. Furthermore, Hsp90 also regulates apoptosis in $C$. albicans via the calcium-calcineurin signaling pathway (Phillips et al., 2003; Lu et al., 2011). Apoptosis is induced by various environmental stimuli, including hydrogen peroxide $\left(\mathrm{H}_{2} \mathrm{O}_{2}\right)$, acetic acid (AA) as well as by drug treatment with AMB in $C$. albicans. Caspase, encoded by the gene CaMCA1, is an essential enzyme involved in apoptosis. Dai et al. demonstrated that compromising Hsp90 inhibits CaMCA1 expression, decreasing caspase activity upon exposure to apoptotic stimuli. Thus, compromised Hsp90 reduces C. albicans apoptosis, partially via down regulation of the calcineurin-caspase signaling pathway (Dai et al., 2012). In conclusion, calcineurin interacts with Hsp90, and the activated calcineurin-Hsp90 complex regulates the stress response, drug resistance, and apoptosis in C. albicans (Juvvadi et al., 2014).

Hsp70 participates in the calcium-calcineurin signaling pathway as well. Msi3, a member of the Hsp70 family, binds Cgr1 during high levels of expression at an early stage of the yeast-hypha transition (Cho et al., 2001, 2003). The relationship between Msi3 and Cgr1 signifies a functional role of Hsp70 in addition to its role in thermal adaptation (Cho et al., 2003). Furthermore, MSI3 and certain calcineurin-dependent genes are highly expressed in response to FLC treatment. Increased Msi3 leads to expression of the calcineurin dependent genes UTR2 and PLC3 in the wide-type strain. However, in the mutant strain tetMsi3, up-regulation of MSI3 is lost in response to FLC treatment, and the expression of calcineurin-dependent genes remains stable. Therefore, induction of calcineurin-dependent gene expression is required for up-regulation of MSI3 expression. These findings illustrate that Msi3 confers FLC resistance in part by activating the calcium-calcineurin signaling pathway. Nagao et al. speculated that Msi3 functions cooperatively with Hsp90 as a cochaperone or through an as yet undiscovered mechanism to activate the calcineurin signaling pathway (Nagao et al., 2012). Thus, the mechanism for Hsp70 regulation of the calcineurin signaling pathway in C. albicans requires further elucidation.

Hsp90 and Hsp70 both participate in the calcium-calcineurin signaling pathway and are crucial to $C$. albicans' drug resistance. Agents targeting calcium, the trigger of Hsp-related calciumcalcineurin signaling show antifungal effects. Inhibitors of calcineurin, cyclosporine A (CsA), and tacrolimus (FK506) inhibit calcineurin by distinct mechanisms (Hemenway and Heitman, 1999). CsA binds with Cpr1, a peptidyl prolyl cis-trans isomerase (cyclophilin A) to calcineurin function. FK506 binds to the structurally unrelated peptidyl-prolyl cis-trans isomerase FKBP12 to block function of calcineurin. Both CsA and FK506 have potent synergistic effects in combination with azoles against C. albicans both in vitro and in vivo (Sun et al., 2008; Uppuluri et al., 2008; Chen et al., 2013). Juvvadi et al. identified a novel serine-proline rich region that is unique to filamentous fungi and absolutely absent in human calcineurin. It has been shown that phosphorylation of the serine-proline rich region regulates the functions of calcineurin in A. fumigatus (Juvvadi et al., 2013). Thus, it is prospective to realize the possibility of inhibiting fungal calcineurin specifically as an antifungal strategy. Additionally, calcium channel blockers (CCB) that disturb cellular calcium homeostasis in C. albicans have synergistic antifungal effects in combination with FLC (Yu et al., 2014a,b; Liu et al., 2016). Yu et al. verified that verapamil inhibits hyphal development and gastrointestinal colonization of C. albicans (Yu et al., 2014a). Furthermore, Liu et al. identified four CCBs, amlodipine, nifedipine, benidipine, and flunarizine, which have synergistic effects with FLC against resistant C. albicans strains (Liu et al., 2016). It may provide a potential therapy to combine CCBs with azoles against $C$. albicans infections in clinic. These discoveries indicate that targeting components of the Hspassociated calcium-calcineurin signaling pathway could exert antifungal characteristics and/or reverse drug tolerance in $C$. albicans.

\section{MAPK Signaling Pathways}

Mitogen activated protein (MAP) kinase signaling pathways are conserved in eukaryotic cells and regulate growth and adaptations stress, including thermal stress, apoptosis, and inflammation. This pathway includes three types of kinases: MAP kinase kinase kinase (MAPKKK), MAP kinase kinase (MAPKK), and MAP kinase (MAPK). When upstream signals act on MAPKKK, MAPKKK is phosphorylated and in turn phosphorylates MAPKK, which in turn phosphorylates MAPK. Ultimately, MAPK signals to downstream transcription factors in order to develop adaptive responses. Thus, MAPK signaling pathways are activated by many extracellular stimuli and mediate signal transduction from the cell surface to the nucleus (Monge et al., 2006). Four MAPK signaling pathways have been identified in C. albicans: the Mkcl pathway, related to cell wall integrity (Navarro-García et al., 1995); the Hog1 pathway, mainly participating in osmotic, oxidative, and other stress adaptions (Smith et al., 2004; Correia et al., 2016); the Cek1 pathway, involved in mating and starvation (Csank et al., 1998; Chen et al., 2002); and the Cek2 pathway, crucial for mating (Chen et al., 2000, 2002). Each of these MAPK signaling pathways plays important roles in the growth and virulence of C. albicans.

MAPK signaling pathways are intimately associated with heat shock responses in C. albicans (Brown et al., 2014). Leach et al. illustrated that $C$. albicans Hsp90 modulates the activity of Hsf1 in response to thermal stress over the short term. In addition, the components of MAPK-Cek1, Hog1, and Mkc1are all client proteins of Hsp90. Compromising Hsp90 function inhibits cell wall biogenesis in C. albicans via impairing activation of Cek1, Hog1, and Mkc1. Thus, Hsp90 modulates long-term thermal adaption via Mkc1-, Hog1-, and Cek1- mediated cell wall remodeling (Leach et al., 2012a; Ene et al., 2015).

Further studies have confirmed that Hsp-associated MAPK signaling pathways contribute to the virulence of $C$. albicans and confer drug tolerance. The Mkcl MAPK pathway is composed of the MAPKKK Bck1, the MAPKK Mkk1 and the terminal transcription factors Swi4/Swi6 (Lafayette et al., 2010; Román et al., 2015). Mediated by protein kinase C ( $\mathrm{Pkc1})$, this pathway 
is responsible for maintaining the integrity of the cell wall during growth, morphogenesis, and cell wall stress in C. albicans (Navarro-García et al., 1995; Lafayette et al., 2010). The cell wall is the first point of contact for antifungal drugs and represents an attractive therapeutic target in fungal pathogens (Cullen and Edgerton, 2016). As mentioned above, Hsp90 interacts with Mkc1, and Hsp90 and Hsp70 help to maintain phosphorylation of activated Pkcl and control the degradation of fully primed and activated Pkc1 (Lum et al., 2013). Therefore, inhibiting Hsp90 both in vitro and in vivo decreases drug resistance to ergosterol biosynthesis inhibitors via destabilization of the terminal MAPK, Mkcl of Pkcl-Mkcl signaling pathway in C. albicans (Singh et al., 2009; Lafayette et al., 2010). Furthermore, LaFayette et al. speculated that the Pkcl-Mkcl and calcineurin pathways, controlled by Hsp90 independently, may regulate drug resistance of C. albicans to ergosterol biosynthesis inhibitors through a single target (Lafayette et al., 2010). In addition, as a member of the Hsp70 family, C. albicans Ssa1 and Ssa2 act as invasins. Ssa1 and Ssa2 combine with host cell cadherins and facilitate host cell endocytosis, allowing C. albicans to invade host cells (Sun et al., 2010). Saraswat et al. suggested that Ssa1 binding with mucin Msb2 detects and regulates thermal stress adaptations, such as survival and hyphae formation at high temperature through the Pkc1-Cek1 signaling pathway (Saraswat et al., 2016). Ssa $1 \Delta / \Delta$ and $M s b 2 \Delta / \Delta$ mutants were shown to exhibit defective phosphorylation of $\mathrm{Mkc1}(\mathrm{P} \sim \mathrm{Mkc1})$ and $\mathrm{P} \sim \mathrm{Cek} 1$, especially at high temperatures. Moreover, some small Hsps are also involved in MAPK signaling. Genetic deletion of HSP21 prevents phosphorylation of Cek1 at elevated temperatures, indicating that phosphorylation of Cek1 during thermal stress is Hsp21dependent (Mayer et al., 2012). In addition, expressions of $C$. albicans HSP12 is regulated by the Hog 1 stress response. It has been shown that devitalized Hog1 represses HSP12 expression. However, stress mediated by activated Hog1 abolishes this repression (Smith et al., 2004; Fu et al., 2012).

These results all verify that Hsps govern the MAPK signaling pathways that play significant roles in the growth and virulence of C. albicans. As a result, inhibiting Hsps or other elements of MAPK signaling should disrupt growth and reduce virulence of C. albicans. Sussman et al. found that cercosporamide, a selective and potent fungal Pkcl kinase inhibitor, acts as a broad-spectrum natural antifungal compound against $C$. albicans. Furthermore, cercosporamide, in combination with an echinocandin analog, exerts synergistic antifungal effects against C. albicans (Sussman et al., 2004). This finding suggests a potential efficient combination strategy to treat $C$. albicans infections. Hopefully, more drugs targeting molecules of the MAPK signaling pathways will be discovered and may help to improve the treatment of C. albicans infections.

\section{RAS1-CAMP-PKA Signaling Pathway}

As a conserved small GTPase on the plasma membrane of eukaryotic cells, Ras regulates a key cyclic Adenosine monophosphate (cAMP)-dependent protein kinase A (PKA) pathway (Mösch et al., 1999; Leberer et al., 2001; Piispanen et al., 2011). In C. albicans, the Ras1-cAMP-PKA signaling pathway is primarily composed of the proteinRas1, adenylate cyclase (AC), cAMP, PKA, and its downstream targets (Hogan and Sundstrom, 2009). Ras1 is described as a "molecular switch," bound to either guanosine triphosphate (GTP) during an activated state by interaction with guanosine nucleotide exchange factors (GEFs) or to guanosine diphosphate (GDP) during inactivated states through interaction with GTPase activator proteins (GAPs) (Fortwendel, 2012). Upon Ras1 activation, Ras1-GTP binds to AC and catalyzes production of the second messenger cAMP. Subsequently, the increase of intracellular cAMP activates cAMP-dependent PKA that phosphorylates a series of transcription factors that control phenotype, metabolism, stress adaption, proliferation, and other functions (Broach, 1991; Rolland et al., 2002; Cassola et al., 2004; Li and Wang, 2013). Further studies show that Efg1, a transcription factor, in parallel with other unidentified signaling molecules, is the terminal point of the Ras1-cAMP-PKA pathway in C. albicans (Bockmühl and Ernst, 2001; Shapiro et al., 2009). Moreover, the Ras1-cAMP-PKA signaling pathway is also involved in normal physiological activities, e.g., acceleration of apoptosis (Phillips et al., 2006), as well as several pathogenic behaviors, such as cell adhesion, hyphal morphogenesis, biofilm formation, and white-to-opaque switching (Inglis and Sherlock, 2013).

Studies have conclusively demonstrated that C. albicans Hsps control growth and virulence of $C$. albicans via interaction with the Ras1-cAMP-PKA pathway (Leberer et al., 2001; Shapiro et al., 2009). Compromising Hsp90 function induces a transition from yeast to hypha growth, while attenuating virulence, in a murine model of $C$. albicans infection. These phenotypes are likely due to $C$. albicans' requirement for morphogenetic flexibility to attain virulence (Shapiro et al., 2009, 2012a). Elevated temperature beyond $37^{\circ} \mathrm{C}$ impairs Hsp90 activity and reverses Hsp90-mediated repression of morphogenetic progress (Shapiro et al., 2012b; Mayer et al., 2013). Shapiro et al. discovered that a $C$. albicans strain with an active RAS1 allele (Ras $\left.1^{V 13}\right)$ and a $C$. albicans strain heterozygous for HSP90 remain as yeast. In contrast, the combination of $R a s 1^{V 13}$ and reduced levels of Hsp90 leads to filamentation. Furthermore, deletion of one IRA2 allele has no effect on Ras1 activity in a strain containing wild type Hsp90; however, deleting one HSP90 allele in the $\operatorname{IRA} 2 \Delta / \Delta$ heterozygote enhances filamentation. In conclusion, genetic epistasis analyses suggest that Hsp90 promotes $C$. albicans morphogenesis by repressing Ras1-cAMP-PKA signaling in a temperature-dependent manner (Shapiro et al., 2009). Other studies have focused on specific interactions between Hsp90 and the Ras1-cAMP-PKA signaling pathway in C. albicans. Shapiro et al. demonstrated that genetic depletion of SGT1 phenocopies compromise of Hsp90 function. They described Sgt1 as a co-chaperone that physically interacts with Hsp90. Moreover, they asserted that Cyr1, the AC of the Ras1-cAMPPKA signaling pathway, interact with Sgt1 and Hsp90, thereby controlling Ras1-cAMP-PKA signaling (Shapiro et al., 2009, 2012 b). We know that tight control of trehalose, regulated by the $G$ protein-coupled receptor (Gpr1), is required for $C$. albicans morphogenesis. Suggesting that trehalose is a possible link between the Ras1-cAMP-PKA pathway and the Hsp90mediated regulation of morphogenesis (Serneels et al., 2012). 
In summary, Hsp90 regulates C. albicans morphogenesis and pathogenesis in response to environmental stress via the Ras1cAMP-PKA signaling pathway. Dodecanol-and farnesol-induced expression of HSP12 stimulates budding growth in wild type C. albicans and is dependent on Ras1-cAMP-PKA signaling (Davis-Hanna et al., 2008; Fu et al., 2012). What's more, Hsp21, which potentiates resistance of $C$. albicans to commonly used antifungal drugs, also lies downstream of the Ras1-cAMPPKA signaling pathway (Harcus et al., 2004; Mayer et al., 2013).

In conclusion, Hsps regulate the virulence properties of $C$. albicans, especially hyphal morphogenesis, via Ras1-cAMP-PKA signaling. Targeting Hsps-related Ras1-cAMP-PKA signaling is also a potential antifungal strategy against $C$. albicans. Jain et al. have shown that MDL-12330A, an inhibitor of mammalian AC, has a synergistic effect in combination with azoles against both azole-susceptible and-resistant strains of $C$. albicans (Jain et al., 2003). In addition, Xie et al. confirmed that staurosporine, a protein kinase inhibitor, abrogates fungal drug resistance via targeting $\mathrm{AC}$ and the cAMP-dependent PKA (Xie et al., 2017). The lichen-derived small molecule retigeric acid $B$ that inhibits AC activity significantly inhibits the filamentation of $C$. albicans. Moreover, it attenuates the virulence of $C$. albicans, leading to prolonged survival in nematodes models (Chang et al., 2012). In short, drugs targeting the Ras1-cAMP-PKA signaling pathway and mechanisms for these drugs need to be further studied for treatment of candidiasis.

\section{Cell Cycle Control Pathways}

In fungi, extracellular and intracellular signals govern the cell cycle, controlling cell division and differentiation. Fungal cells exhibit different morphologies, affected by the activity of cyclin-dependent kinases. Cell cycle regulation is pivotal for the virulence and infectious development of fungal pathogens (Berman, 2006; Pérez-Martín et al., 2016).

Leach et al. observed that Hsf1 and Hsp90 orchestrate temperature-dependent global transcriptional remodeling of chromatin architecture in C. albicans, indicating Hsps may participate in movement of the cell nucleus (Leach et al., 2016). Further studies suggest that Hsps are involved in the cell cycle control pathways of $C$. albicans. Senn et al. found that filaments of C. albicans induced by compromised Hsp90 are neither pseudohyphae nor hyphae-like, but rather are similar to filaments induced by cell cycle arrest (Senn et al., 2012). This finding implies that Hsp90 is involved in cell cycle control and affects morphogenesis of $C$. albicans. Further studies illustrate that Cdc28, a kind of cyclin-dependent kinase in C. albicans, is normally stable, but its depletion leads to filamentous growth (Umeyama et al., 2006). However, levels of Cdc28 are markedly reduced in response to Hsp90 depletion, while CDC28 transcript levels remain stable. Therefore, reduced levels of Hsp90 may result in instability of Cdc28. Thus, Senn et al. speculated that Cdc28 is a candidate for an Hsp90 client protein and is one of the targets of Hsp90's influence on cell cycle control in $C$. albicans (Senn et al., 2012). Additionally, Hsp90 is required for the stability of two C. albicans mitotic cyclins, Clb2, and Clb4
(Bensen et al., 2005). Nevertheless, CLB deletion does not lead to Hsp90 inhibition, and so it is less likely to contribute to Hsp90's impacts on morphogenesis (Bensen et al., 2005; Senn et al., 2012). Moreover, Shapiro et al. showed that Hms1, a transcriptional regulator, contributes to morphogenesis induced by elevated temperature or impaired $\mathrm{Hsp} 90$ in C. albicans. Hms1 lies downstream of the cyclin Pcl1 and the cyclindependent kinase Pho85. In response to inhibition of Hsp90, Hms1 promotes filamentous growth by activating the expression of UME6 and RBT5. Meanwhile, deleting HMS1 improves the survival rates of $C$. albicans infection in metazoan models (Shapiro et al., 2012a). These data indicate a potential antifungal strategy for treating $C$. albicans infections by targeting elements in the Pho85-Pcl1-Hms1 pathway. These findings demonstrate that Hsp90 governs morphogenesis via multiple elements of cell cycle control pathways in C. albicans. Thus, modulation of Hsp90 is a promising antifungal strategy to treat C. albicans infections.

\section{Others}

Other signaling pathways in C. albicans have been shown to be regulated by Hsps. Leach et al. first revealed a molecular link between membrane fluidity and the heat shock response (Leach and Cowen, 2014). In response to elevated temperature, the E3 ubiquitin ligase Rsp5, which controls expression of OLE1 via transcription factor Spt23, is a hypothesized early sensor of temperature. The OLE1 gene encodes a fatty acid desaturase, and decrease of OLE1 triggers expression of FAS2, which encodes a fatty acid synthase. Moreover, the decrease of OLE1 prevents activation of Hsf1 and reduces expression of HSP in response to heat shock. This finding confirms the link between membrane fluidity and the heat shock response. In addition, OLE1 not only controls levels of fatty acid desaturase, which regulates fluidity of $C$. albicans membrane, but also facilitates hyphae development, a crucial process for C. albicans virulence (Krishnamurthy et al., 2004; Noverr and Huffnagle, 2004). Thus, disrupting Hsp-regulated membrane fluidity is a potential antifungal strategy against $C$. albicans.

Robbins et al. reported that impairing Hsp90 governs dispersion and reverses antifungal drug tolerance of C. albicans biofilms via down-regulation of matrix glucan (Robbins et al., 2011). They illustrated that Hsp90 regulates glucan levels either by affecting Fks1, an important synthase for the production of matrix glucan, or by affecting Zap1 and its downstream targets Gca1 and Gca2, controlling the hydrolytic release of $\beta$-glucan fragments to the matrix. Existence of matrix glucan is a barrier for drugs in reaching $C$. albicans and promotes its invasive ability. Therefore, targeting matrix glucan via Hsp90 disruption may also be a good therapeutic strategy against C. albicans biofilms.

\section{CONCLUSION}

Initially, Hsps in C. albicans were considered simply a series of proteins generated in response to thermal stress. Further studies suggest that in addition to regulating thermal adaptations, Hsps 

in diverse signaling pathways, such as the calcium-calcineurin signaling pathway, MAPK signaling pathways, the Ras1-cAMPPKA signaling pathway and cell cycle control pathways. These Hsp-associated pathways are essential for controlling basic physiological activities and virulence of $C$. albicans. Further studies have shown that agents targeting Hsps and elements of Hsp-associated pathways exert antifungal effects and/or reverse tolerance of $C$. albicans to traditional antifungal drugs. These findings support the hypothesis that understanding the link between Hsps and its many signaling partners could lead to the elucidation of several novel antifungal targets to be explored in $C$. albicans. act as molecular chaperones that interact with many molecules

\section{AUTHOR CONTRIBUTIONS}

YG wrote the review and created Figure 1 and Table 1, SS, CY, and TL helped with it.

\section{FUNDING}

This work was supported by grants from the Department of Science and Technology of Shandong Province of China (2013GSF11848 and 2016GSF201187) and Shandong Provincial Natural Science Foundation of China (2015GSF121052). The funders had no role in the study design, data collection, and analysis, decision to publish, or preparation of the manuscript.

Cho, T., Sudoh, M., Tanaka, T., Nakashima, Y., Chibana, H., and Kaminishi, H. (2001). Isolation and expression of a gene (CGR1) regulated during the yeasthyphal transition in Candida albicans. Biochim. Biophys. Acta 1517, 288-292. doi: 10.1016/S0167-4781(00)00243-8

Becherelli, M., Tao, J., and Ryder, N. S. (2013). Involvement of heat shock proteins in Candida albicans biofilm formation. J. Mol. Microbiol. Biotechnol. 23, 396-400. doi: 10.1159/000351619

Bensen, E. S., Clemente-Blanco, A., Finley, K. R., Correa-Bordes, J., and Berman, J. (2005). The mitotic cyclins Clb2p and Clb4p affect morphogenesis in Candida albicans. Mol. Biol. Cell 16, 3387-3400. doi: 10.1091/mbc.E04-12-1081

Berman, J. (2006). Morphogenesis and cell cycle progression in Candida albicans. Curr. Opin. Microbiol. 9, 595-601. doi: 10.1016/j.mib.2006.10.007

Bockmühl, D. P., and Ernst, J. F. (2001). A potential phosphorylation site for an Atype kinase in the Efg1 regulator protein contributes to hyphal morphogenesis of Candida albicans. Genetics 157, 1523-1530.

Broach, J. R. (1991). Ras-regulated signaling processes in Saccharomyces cerevisiae. Curr. Opin. Genet. Dev. 1, 370-377. doi: 10.1016/S0959-437X(05)80302-8

Brown, A. J., Budge, S., Kaloriti, D., Tillmann, A., Jacobsen, M. D., Yin, Z., et al. (2014). Stress adaptation in a pathogenic fungus. J. Exp. Biol. 217, 144-155. doi: $10.1242 / \mathrm{jeb} .088930$

Bugli, F., Cacaci, M., Martini, C., Torelli, R., Posteraro, B., Sanguinetti, M., et al. (2013). Human monoclonal antibody-based therapy in the treatment of invasive candidiasis. Clin. Dev. Immunol. 2013:403121. doi: $10.1155 / 2013 / 403121$

Burnie, J. P., Carter, T. L., Hodgetts, S. J., and Matthews, R. C. (2006). Fungal heat-shock proteins in human disease. FEMS Microbiol. Rev. 30, 53-88. doi: 10.1111/j.1574-6976.2005.00001.x

Cassola, A., Parrot, M., Silberstein, S., Magee, B. B., Passeron, S., Giasson, L., et al. (2004). Candida albicans lacking the gene encoding the regulatory subunit of protein kinase A displays a defect in hyphal formation and an altered localization of the catalytic subunit. Eukaryotic Cell 3, 190-199. doi: 10.1128/EC.3.1.190-199.2004

Chang, W., Li, Y., Zhang, L., Cheng, A., and Lou, H. (2012). Retigeric acid B attenuates the virulence of Candida albicans via inhibiting adenylyl cyclase activity targeted by enhanced farnesol production. PLoS ONE 7:e41624. doi: 10.1371/journal.pone.0041624

Chatterjee, S., and Tatu, U. (2017). Heat shock protein 90 localizes to the surface and augments virulence factors of Cryptococcus neoformans. PLoS Negl. Trop. Dis. 11:e005836. doi: 10.1371/journal.pntd.0005836

Chen, J., Chen, J., Lane, S., and Liu, H. (2002). A conserved mitogen-activated protein kinase pathway is required for mating in Candida albicans. Mol. Microbiol. 46, 1335-1344. doi: 10.1046/j.1365-2958.2002.03249.x

Chen, J., Wang, Q., and Chen, J. Y. (2000). CEK2, a novel MAPK from Candida albicans complement the mating defect of fus3/kss1 Mutant. Sheng Wu Hua Xue Yu Sheng Wu Wu Li Xue Bao 32, 299-304.

Chen, Y. L., Lehman, V. N., Averette, A. F., Perfect, J. R., and Heitman, J. (2013). Posaconazole exhibits in vitro and in vivo synergistic antifungal activity with caspofungin or FK506 against Candida albicans. PLoS ONE 8:e57672. doi: 10.1371/journal.pone.0057672
Cho, T., Toyoda, M., Sudoh, M., Nakashima, Y., Calderone, R. A., and Kaminishi, H. (2003). Isolation and sequencing of the Candida albicans MSI3, a putative novel member of the HSP70 family. Yeast 20, 149-156. doi: 10.1002/yea.952

Cordeiro Rde, A., Evangelista, A. J., Serpa, R., Marques, F. J., de Melo, C. V., de Oliveira, J. S., et al. (2016). Inhibition of heat-shock protein 90 enhances the susceptibility to antifungals and reduces the virulence of Cryptococcus neoformans/Cryptococcus gattii species complex. Microbiology 162, 309-317. doi: $10.1099 /$ mic. 0.000222

Correia, I., Alonso-Monge, R., and Pla, J. (2016). The Hog1 MAP kinase promotes the recovery from cell cycle arrest induced by hydrogen peroxide in Candida albicans. Front Microbiol 7:2133. doi: 10.3389/fmicb.2016.02133

Cowen, L. E. (2009). Hsp90 orchestrates stress response signaling governing fungal drug resistance. PLoS Pathog. 5:e1000471. doi: 10.1371/journal.ppat.1000471

Cowen, L. E., and Lindquist, S. (2005). Hsp90 potentiates the rapid evolution of new traits: drug resistance in diverse fungi. Science 309, 2185-2189. doi: $10.1126 /$ science. 1118370

Cowen, L. E., Carpenter, A. E., Matangkasombut, O., Fink, G. R., and Lindquist, S. (2006). Genetic architecture of Hsp90-dependent drug resistance. Eukaryot Cell 5, 2184-2188. doi: 10.1128/EC.00274-06

Cowen, L. E., Singh, S. D., Köhler, J. R., Collins, C., Zaas, A. K., Schell, W. A., et al. (2009). Harnessing Hsp90 function as a powerful, broadly effective therapeutic strategy for fungal infectious disease. Proc. Natl. Acad. Sci. U.S.A. 106, 2818-2823. doi: 10.1073/pnas.0813394106

Craig, E. A., Gambill, B. D., and Nelson, R. J. (1993). Heat shock proteins: molecular chaperones of protein biogenesis. Microbiol. Rev. 57, 402-414.

Cruz, M. C., Goldstein, A. L., Blankenship, J. R., Del Poeta, M., Davis, D., Cardenas, M. E., et al. (2002). Calcineurin is essential for survival during membrane stress in Candida albicans. EMBO J. 21, 546-559. doi: 10.1093/emboj/21. 4.546

Csank, C., Schröppel, K., Leberer, E., Harcus, D., Mohamed, O., Meloche, S., et al. (1998). Roles of the Candida albicans mitogen-activated protein kinase homolog, Ceklp, in hyphal development and systemic candidiasis. Infect. Immun. 66, 2713-2721.

Cuéllar-Cruz, M., López-Romero, E., Ruiz-Baca, E., and Zazueta-Sandoval, R. (2014). Differential response of Candida albicans and Candida glabrata to oxidative and nitrosative stresses. Curr. Microbiol. 69, 733-739. doi: 10.1007/s00284-014-0651-3

Cuenca-Estrella, M. (2014). Antifungal drug resistance mechanisms in pathogenic fungi: from bench to bedside. Clin. Microbiol. Infect. 20(Suppl 6), 54-59. doi: 10.1111/1469-0691.12495

Cullen, P. J., and Edgerton, M. (2016). Unmasking fungal pathogens by studying MAPK-dependent cell wall regulation in Candida albicans. Virulence 7, 502-505. doi: 10.1080/21505594.2016.1177695 
Dai, B., Wang, Y., Li, D., Xu, Y., Liang, R., Zhao, L., et al. (2012). Hsp90 is involved in apoptosis of Candida albicans by regulating the calcineurin-caspase apoptotic pathway. PLoS ONE 7:e45109. doi: 10.1371/journal.pone.0045109

Davis-Hanna, A., Piispanen, A. E., Stateva, L. I., and Hogan, D. A. (2008). Farnesol and dodecanol effects on the Candida albicans Ras1-cAMP signalling pathway and the regulation of morphogenesis. Mol. Microbiol. 67, 47-62. doi: 10.1111/j.1365-2958.2007.06013.x

Dimopoulos, G., Karabinis, A., Samonis, G., and Falagas, M. E. (2007). Candidemia in immunocompromised and immunocompetent critically ill patients: a prospective comparative study. Eur. J. Clin. Microbiol. Infect. Dis. 26, 377-384. doi: 10.1007/s10096-007-0316-2

Ene, I. V., Walker, L. A., Schiavone, M., Lee, K. K., Martin-Yken, H., Dague, E., et al. (2015). Cell wall remodeling enzymes modulate fungal cell wall elasticity and osmotic stress resistance. MBio 6:e00986. doi: 10.1128/mBio.00986-15

Enjalbert, B., Nantel, A., and Whiteway, M. (2003). Stress-induced gene expression in Candida albicans: absence of a general stress response. Mol. Biol. Cell 14, 1460-1467. doi: 10.1091/mbc.E02-08-0546

Eroles, P., Sentandreu, M., Elorza, M. V., and Sentandreu, R. (1997). The highly immunogenic enolase and Hsp70p are adventitious Candida albicans cell wall proteins. Microbiology 143(Pt 2), 313-320. doi: 10.1099/00221287-143-2-313

Fiori, A., Kucharíková, S., Govaert, G., Cammue, B. P., Thevissen, K., and Van Dijck, P. (2012). The heat-induced molecular disaggregase Hsp104 of Candida albicans plays a role in biofilm formation and pathogenicity in a worm infection model. Eukaryot Cell 11, 1012-1020. doi: 10.1128/EC.00147-12

Fortwendel, J. R. (2012). Ras-mediated signal transduction and virulence in human pathogenic fungi. Fungal Genome Biol. 2:105. doi: 10.4172/2165-8056.1000105

Fu, M. S., De Sordi, L., and Mühlschlegel, F. A. (2012). Functional characterization of the small heat shock protein Hsp12p from Candida albicans. PLoS ONE 7:e42894. doi: 10.1371/journal.pone.0042894

Glover, J. R., and Lindquist, S. (1998). Hsp104, Hsp70, and Hsp40: a novel chaperone system that rescues previously aggregated proteins. Cell 94, 73-82. doi: 10.1016/S0092-8674(00)81223-4

Habich, C., Kempe, K., Gomez, F. J., Lillicrap, M., Gaston, H., van der Zee, R., et al. (2006). Heat shock protein 60: identification of specific epitopes for binding to primary macrophages. FEBS Lett. 580, 115-120. doi: 10.1016/j.febslet.2005.11.060

Harcus, D., Nantel, A., Marcil, A., Rigby, T., and Whiteway, M. (2004). Transcription profiling of cyclic AMP signaling in Candida albicans. Mol. Biol. Cell 15, 4490-4499. doi: 10.1091/mbc.E04-02-0144

Heath, V. L., Shaw, S. L., Roy, S., and Cyert, M. S. (2004). Hph1p and Hph2p, novel components of calcineurin-mediated stress responses in Saccharomyces cerevisiae. Eukaryot Cell 3, 695-704. doi: 10.1128/EC.3.3.695-704.2004

Hemenway, C. S., and Heitman, J. (1999). Calcineurin. Structure, function, and inhibition. Cell Biochem. Biophys. 30, 115-151. doi: 10.1007/BF02737887

Hodgetts, S., Nooney, L., Al-Akeel, R., Curry, A., Awad, S., Matthews, R., et al. (2008). Efungumab and caspofungin: pre-clinical data supporting synergy. J. Antimicrob. Chemother. 61, 1132-1139. doi: 10.1093/jac/dkn075

Hogan, D. A., and Sundstrom, P. (2009). The Ras/cAMP/PKA signaling pathway and virulence in Candida albicans. Future Microbiol. 4, 1263-1270. doi: $10.2217 / \mathrm{fmb} .09 .106$

Inglis, D. O., and Sherlock, G. (2013). Ras signaling gets fine-tuned: regulation of multiple pathogenic traits of Candida albicans. Eukaryot Cell 12, 1316-1325. doi: 10.1128/EC.00094-13

Jackson, S. E. (2013). Hsp90: structure and function. Top. Curr. Chem. 328, 155-240. doi: 10.1007/128_2012_356

Jain, P., Akula, I., and Edlind, T. (2003). Cyclic AMP signaling pathway modulates susceptibility of candida species and Saccharomyces cerevisiae to antifungal azoles and other sterol biosynthesis inhibitors. Antimicrob. Agents Chemother. 47, 3195-3201. doi: 10.1128/AAC.47.10.3195-3201.2003

Jaya, N., Garcia, V., and Vierling, E. (2009). Substrate binding site flexibility of the small heat shock protein molecular chaperones. Proc. Natl. Acad. Sci. U.S.A. 106, 15604-15609. doi: 10.1073/pnas.0902177106

Juvvadi, P. R., Gehrke, C., Fortwendel, J. R., Lamoth, F., Soderblom, E. J., Cook, E. C., et al. (2013). Phosphorylation of Calcineurin at a novel serine-proline rich region orchestrates hyphal growth and virulence in Aspergillus fumigatus. PLoS Pathog. 9:e1003564. doi: 10.1371/journal.ppat.1003564

Juvvadi, P. R., Lamoth, F., and Steinbach, W. J. (2014). Calcineurin as a multifunctional regulator: unraveling novel functions in fungal stress responses, hyphal growth, drug resistance, and pathogenesis. Fungal Biol. Rev. 28, 56-69. doi: 10.1016/j.fbr.2014.02.004

Karababa, M., Valentino, E., Pardini, G., Coste, A. T., Bille, J., and Sanglard, D. (2006). CRZ1, a target of the calcineurin pathway in Candida albicans. Mol. Microbiol. 59, 1429-1451. doi: 10.1111/j.1365-2958.2005.05037.x

Karwa, R., and Wargo, K. A. (2009). Efungumab: a novel agent in the treatment of invasive candidiasis. Ann. Pharmacother. 43, 1818-1823. doi: 10.1345/aph.1M218

Kim, Y. S., Alarcon, S. V., Lee, S., Lee, M. J., Giaccone, G., Neckers, L., et al. (2009). Update on Hsp90 inhibitors in clinical trial. Curr. Top. Med. Chem. 9, 1479-1492. doi: 10.2174/156802609789895728

Kraus, P. R., and Heitman, J. (2003). Coping with stress: calmodulin and calcineurin in model and pathogenic fungi. Biochem. Biophys. Res. Commun. 311, 1151-1157. doi: 10.1016/S0006-291X(03)01528-6

Kriengkauykiat, J., Ito, J. I., and Dadwal, S. S. (2011). Epidemiology and treatment approaches in management of invasive fungal infections. Clin. Epidemiol. 3, 175-191. doi: 10.2147/CLEP.S12502

Krishnamurthy, S., Plaine, A., Albert, J., Prasad, T., Prasad, R., and Ernst, J. F. (2004). Dosage-dependent functions of fatty acid desaturase Olelp in growth and morphogenesis of Candida albicans. Microbiology 150, 1991-2003. doi: 10.1099/mic.0.27029-0

Lafayette, S. L., Collins, C., Zaas, A. K., Schell, W. A., Betancourt-Quiroz, M., Gunatilaka, A. A., et al. (2010). PKC signaling regulates drug resistance of the fungal pathogen Candida albicans via circuitry comprised of $\mathrm{Mkc1}$, calcineurin, and Hsp90. PLoS Pathog. 6:e1001069. doi: 10.1371/journal.ppat.1001069

Lamoth, F., Juvvadi, P. R., and Steinbach, W. J. (2016). Heat shock protein 90 (Hsp90): a novel antifungal target against Aspergillus fumigatus. Crit. Rev. Microbiol. 42, 310-321. doi: 10.3109/1040841X.2014.947239

Leach, M. D., and Cowen, L. E. (2014). Membrane fluidity and temperature sensing are coupled via circuitry comprised of Ole1, Rsp5, and Hsf1 in Candida albicans. Eukaryot Cell 13, 1077-1084. doi: 10.1128/EC.00138-14

Leach, M. D., Budge, S., Walker, L., Munro, C., Cowen, L. E., and Brown, A. J. (2012a). Hsp90 orchestrates transcriptional regulation by Hsf1 and cell wall remodelling by MAPK signalling during thermal adaptation in a pathogenic yeast. PLoS Pathog. 8:e1003069. doi: 10.1371/journal.ppat.10 03069

Leach, M. D., Farrer, R. A., Tan, K., Miao, Z., Walker, L. A., Cuomo, C. A., et al. (2016). Hsf1 and Hsp90 orchestrate temperature-dependent global transcriptional remodelling and chromatin architecture in Candida albicans. Nat. Commun. 7:11704. doi: 10.1038/ncomms11704

Leach, M. D., Klipp, E., Cowen, L. E., and Brown, A. J. (2012b). Fungal Hsp90: a biological transistor that tunes cellular outputs to thermal inputs. Nat. Rev. Microbiol. 10, 693-704. doi: 10.1038/nrmicro2875

Leberer, E., Harcus, D., Dignard, D., Johnson, L., Ushinsky, S., Thomas, D. Y., et al. (2001). Ras links cellular morphogenesis to virulence by regulation of the MAP kinase and cAMP signalling pathways in the pathogenic fungus Candida albicans. Mol. Microbiol. 42, 673-687. doi: 10.1046/j.1365-2958.2001.0 2672.x

Léger, T., Garcia, C., Ounissi, M., Lelandais, G., and Camadro, J. M. (2015). The metacaspase (Mcalp) has a dual role in farnesol-induced apoptosis in Candida albicans. Mol. Cell. Proteomics 14, 93-108. doi: 10.1074/mcp.M114.041210

Li, L., An, M., Shen, H., Huang, X., Yao, X., Liu, J., et al. (2015). The non-Geldanamycin Hsp90 inhibitors enhanced the antifungal activity of fluconazole. Am. J. Transl. Res. 7, 2589-2602.

Li, X. S., Sun, J. N., Okamoto-Shibayama, K., and Edgerton, M. (2006). Candida albicans cell wall ssa proteins bind and facilitate import of salivary histatin 5 required for toxicity. J. Biol. Chem. 281, 22453-22463. doi: 10.1074/jbc.M604064200

Li, X., and Sun, S. (2016). Targeting the fungal calcium-calcineurin signaling network in overcoming drug resistance. Future Med. Chem. 8, 1379-1381. doi: 10.4155/fmc-2016-0094

Li, X., Robbins, N., O'Meara, T. R., and Cowen, L. E. (2017). Extensive functional redundancy in the regulation of Candida albicans drug resistance and morphogenesis by lysine deacetylases Hos2, Hda1, Rpd3 and Rpd31. Mol. Microbiol. 103, 635-656. doi: 10.1111/mmi.13578

$\mathrm{Li}, \mathrm{Y}$., and Wang, Y. (2013). Ras protein/cAMP-dependent protein kinase signaling is negatively regulated by a deubiquitinating enzyme, Ubp3, in yeast. J. Biol. Chem. 288, 11358-11365. doi: 10.1074/jbc.M112.449751 
Liu, S., Hou, Y., Liu, W., Lu, C., Wang, W., and Sun, S. (2015). Components of the calcium-calcineurin signaling pathway in fungal cells and their potential as antifungal targets. Eukaryot. Cell 14, 324-334. doi: 10.1128/EC.00271-14

Liu, S., Yue, L., Gu, W., Li, X., Zhang, L., and Sun, S. (2016). Synergistic effect of fluconazole and calcium channel blockers against resistant Candida albicans. PLoS ONE 11:e0150859. doi: 10.1371/journal.pone.0168936

López-Ribot, J. L., Alloush, H. M., Masten, B. J., and Chaffin, W. L. (1996). Evidence for presence in the cell wall of Candida albicans of a protein related to the hsp70 family. Infect. Immun. 64, 3333-3340.

Louie, A., Stein, D. S., Zack, J. Z., Liu, W., Conde, H., Fregeau, C., et al. (2011). Dose range evaluation of Mycograb C28Y variant, a human recombinant antibody fragment to heat shock protein 90, in combination with amphotericin Bdesoxycholate for treatment of murine systemic candidiasis. Antimicrob. Agents Chemother. 55, 3295-3304. doi: 10.1128/AAC.01324-10

Lu, H., Zhu, Z., Dong, L., Jia, X., Sun, X., Yan, L., et al. (2011). Lack of trehalose accelerates $\mathrm{H} 2 \mathrm{O} 2$-induced Candida albicans apoptosis through regulating Ca2+ signaling pathway and caspase activity. PLOS ONE 6:e15808. doi: 10.1371/journal.pone.0015808

Lum, M. A., Balaburski, G. M., Murphy, M. E., Black, A. R., and Black, J. D. (2013). Heat shock proteins regulate activation-induced proteasomal degradation of the mature phosphorylated form of protein kinase C. J. Biol. Chem. 288, 27112-27127. doi: 10.1074/jbc.M112.437095

Maneu, V., Cervera, A. M., Martinez, J. P., and Gozalbo, D. (1997). Molecular cloning of a Candida albicans gene (SSB1) coding for a protein related to the Hsp70 family. Yeast 13, 677-681. doi: 10.1002/(SICI)10970061(19970615)13:7<677::AID-YEA131>3.0.CO;2-6

Maneu, V., Roig, P., and Gozalbo, D. (2000). Complementation of Saccharomyces cerevisiae mutations in genes involved in translation and protein folding (EFB1 and SSB1) with Candida albicans cloned genes. Res. Microbiol. 151, 739-746. doi: 10.1016/S0923-2508(00)01139-6

Martínez-Ruiz, A., Villanueva, L., González de Orduña, C., López-Ferrer, D., Higueras, M. A., Tarín, C., et al. (2005). S-nitrosylation of Hsp90 promotes the inhibition of its ATPase and endothelial nitric oxide synthase regulatory activities. Proc. Natl. Acad. Sci. U.S.A. 102, 8525-8530. doi: $10.1073 /$ pnas. 0407294102

Matthews, R. C., Burnie, J. P., and Tabaqchali, S. (1984). Immunoblot analysis of the serological response in systemic candidosis. Lancet 2, 1415-1418. doi: 10.1016/S0140-6736(84)91618-0

Matthews, R. C., Burnie, J. P., and Tabaqchali, S. (1987). Isolation of immunodominant antigens from sera of patients with systemic candidiasis and characterization of serological response to Candida albicans. J. Clin. Microbiol. $25,230-237$.

Matthews, R. C., Rigg, G., Hodgetts, S., Carter, T., Chapman, C., Gregory, C., et al. (2003). Preclinical assessment of the efficacy of mycograb, a human recombinant antibody against fungal HSP90. Antimicrob. Agents Chemother. 47, 2208-2216. doi: 10.1128/AAC.47.7.2208-2216.2003

Mayer, F. L., Wilson, D., and Hube, B. (2013). Hsp21 potentiates antifungal drug tolerance in Candida albicans. PLoS ONE 8:e60417. doi: 10.1371/journal.pone.0060417

Mayer, F. L., Wilson, D., Jacobsen, I. D., Miramón, P., Slesiona, S., Bohovych, I. M., et al. (2012). Small but crucial: the novel small heat shock protein Hsp21 mediates stress adaptation and virulence in Candida albicans. PLoS ONE 7:e38584. doi: 10.1371/journal.pone.0038584

Monge, R. A., Román, E., Nombela, C., and Pla, J. (2006). The MAP kinase signal transduction network in Candida albicans. Microbiology 152, 905-912. doi: 10.1099/mic.0.28616-0

Mösch, H. U., Kübler, E., Krappmann, S., Fink, G. R., and Braus, G. H. (1999). Crosstalk between the Ras2p-controlled mitogen-activated protein kinase and cAMP pathways during invasive growth of Saccharomyces cerevisiae. Mol. Biol. Cell 10, 1325-1335. doi: 10.1091/mbc.10.5.1325

Nagao, J., Cho, T., Uno, J., Ueno, K., Imayoshi, R., Nakayama, H., et al. (2012). Candida albicans Msi3p, a homolog of the Saccharomyces cerevisiae Sselp of the Hsp70 family, is involved in cell growth and fluconazole tolerance. FEMS Yeast Res. 12, 728-737. doi: 10.1111/j.1567-1364.2012.00822.x

Navarro-García, F., Sánchez, M., Pla, J., and Nombela, C. (1995). Functional characterization of the MKC1 gene of Candida albicans, which encodes a mitogen-activated protein kinase homolog related to cell integrity. Mol. Cell. Biol. 15, 2197-2206. doi: 10.1128/MCB.15.4.2197
Nicholls, S., Leach, M. D., Priest, C. L., and Brown, A. J. (2009). Role of the heat shock transcription factor, Hsf1, in a major fungal pathogen that is obligately associated with warm-blooded animals. Mol. Microbiol. 74, 844-861. doi: $10.1111 /$ j.1365-2958.2009.06883.x

Noverr, M. C., and Huffnagle, G. B. (2004). Regulation of Candida albicans morphogenesis by fatty acid metabolites. Infect. Immun. 72, 6206-6210. doi: 10.1128/IAI.72.11.6206-6210.2004

Odds, F. C., Brown, A. J., and Gow, N. A. (2003). Antifungal agents: mechanisms of action. Trends Microbiol. 11, 272-279. doi: 10.1016/S0966-842X(03)0 0117-3

O'meara, T. R., and Cowen, L. E. (2014). Hsp90-dependent regulatory circuitry controlling temperature-dependent fungal development and virulence. Cell. Microbiol. 16, 473-481. doi: 10.1111/cmi.12266

Pachl, J., Svoboda, P., Jacobs, F., Vandewoude, K., van der Hoven, B., Spronk, P., et al. (2006). A randomized, blinded, multicenter trial of lipid-associated amphotericin B alone versus in combination with an antibody-based inhibitor of heat shock protein 90 in patients with invasive candidiasis. Clin. Infect. Dis. 42, 1404-1413. doi: 10.1086/503428

Pérez-Martín, J., Bardetti, P., Castanheira, S., De La Torre, A., and TenorioGómez, M. (2016). Virulence-specific cell cycle and morphogenesis connections in pathogenic fungi. Semin. Cell Dev. Biol. 57, 93-99. doi: 10.1016/j.semcdb.2016.03.017

Pfaller, M. A., Messer, S. A., Georgopapadakou, N., Martell, L. A., Besterman, J. M., and Diekema, D. J. (2009). Activity of MGCD290, a Hos2 histone deacetylase inhibitor, in combination with azole antifungals against opportunistic fungal pathogens. J. Clin. Microbiol. 47, 3797-3804. doi: 10.1128/JCM.00618-09

Phillips, A. J., Crowe, J. D., and Ramsdale, M. (2006). Ras pathway signaling accelerates programmed cell death in the pathogenic fungus Candida albicans. Proc. Natl. Acad. Sci. U.S.A. 103, 726-731. doi: 10.1073/pnas.0506405103

Phillips, A. J., Sudbery, I., and Ramsdale, M. (2003). Apoptosis induced by environmental stresses and amphotericin B in Candida albicans. Proc. Natl. Acad. Sci. U.S.A. 100, 14327-14332. doi: 10.1073/pnas.2332326100

Piispanen, A. E., Bonnefoi, O., Carden, S., Deveau, A., Bassilana, M., and Hogan, D. A. (2011). Roles of Ras1 membrane localization during Candida albicans hyphal growth and farnesol response. Eukaryot. Cell 10, 1473-1484. doi: 10.1128/EC.05153-11

Powers, M. V., and Workman, P. (2007). Inhibitors of the heat shock response: biology and pharmacology. FEBS Lett. 581, 3758-3769. doi: 10.1016/j.febslet.2007.05.040

Raggam, R. B., Salzer, H. J., Marth, E., Heiling, B., Paulitsch, A. H., and Buzina, W. (2011). Molecular detection and characterisation of fungal heat shock protein 60. Mycoses 54, e394-e399. doi: 10.1111/j.1439-0507.2010.01933.x

Rajaiah, R., and Moudgil, K. D. (2009). Heat-shock proteins can promote as well as regulate autoimmunity. Autoimmun. Rev. 8, 388-393. doi: 10.1016/j.autrev.2008.12.004

Retzlaff, M., Stahl, M., Eberl, H. C., Lagleder, S., Beck, J., Kessler, H., et al. (2009). Hsp90 is regulated by a switch point in the C-terminal domain. EMBO Rep. 10, 1147-1153. doi: 10.1038/embor.2009.153

Richie, D. L., Ghannoum, M. A., Isham, N., Thompson, K. V., and Ryder, N. S. (2012). Nonspecific effect of Mycograb on amphotericin B MIC. Antimicrob. Agents Chemother. 56, 3963-3964. doi: 10.1128/AAC.00435-12

Robbins, N., Leach, M. D., and Cowen, L. E. (2012). Lysine deacetylases Hdal and Rpd3 regulate Hsp90 function thereby governing fungal drug resistance. Cell Rep. 2, 878-888. doi: 10.1016/j.celrep.2012.08.035

Robbins, N., Uppuluri, P., Nett, J., Rajendran, R., Ramage, G., Lopez-Ribot, J. L., et al. (2011). Hsp90 governs dispersion and drug resistance of fungal biofilms. PLoS Pathog. 7:e1002257. doi: 10.1371/journal.ppat.1002257

Rolland, F., Winderickx, J., and Thevelein, J. M. (2002). Glucose-sensing and -signalling mechanisms in yeast. FEMS Yeast Res. 2, 183-201. doi: 10.1111/j.1567-1364.2002.tb00084.x

Román, E., Alonso-Monge, R., Miranda, A., and Pla, J. (2015). The Mkk2 MAPKK regulates cell wall biogenesis in cooperation with the Cek1-Pathway in Candida albicans. PLoS ONE 10:e0133476. doi: 10.1371/journal.pone.0133476

Sanchez, Y., and Lindquist, S. L. (1990). HSP104 required for induced thermotolerance. Science 248, 1112-1115. doi: 10.1126/science.2188365

Saraswat, D., Kumar, R., Pande, T., Edgerton, M., and Cullen, P. J. (2016). Signalling mucin Msb2 Regulates adaptation to thermal stress in Candida albicans. Mol. Microbiol. 100, 425-441. doi: 10.1111/mmi.13326 
Senn, H., Shapiro, R. S., and Cowen, L. E. (2012). Cdc28 provides a molecular link between Hsp90, morphogenesis, and cell cycle progression in Candida albicans. Mol. Biol. Cell 23, 268-283. doi: 10.1091/mbc.E11-08-0729

Serneels, J., Tournu, H., and Van Dijck, P. (2012). Tight control of trehalose content is required for efficient heat-induced cell elongation in Candida albicans. J. Biol. Chem. 287, 36873-36882. doi: 10.1074/jbc.M112.402651

Shapiro, R. S., Sellam, A., Tebbji, F., Whiteway, M., Nantel, A., and Cowen, L. E. (2012a). Pho85, Pcl1, and Hms1 signaling governs Candida albicans morphogenesis induced by high temperature or Hsp90 compromise. Curr. Biol. 22, 461-470. doi: 10.1016/j.cub.2012.01.062

Shapiro, R. S., Uppuluri, P., Zaas, A. K., Collins, C., Senn, H., Perfect, J. R., et al. (2009). Hsp90 orchestrates temperature-dependent Candida albicans morphogenesis via Ras1-PKA signaling. Curr. Biol. 19, 621-629. doi: 10.1016/j.cub.2009.03.017

Shapiro, R. S., Zaas, A. K., Betancourt-Quiroz, M., Perfect, J. R., and Cowen, L. E. (2012b). The Hsp90 co-chaperone Sgtl governs Candida albicans morphogenesis and drug resistance. PLoS ONE 7:e44734. doi: 10.1371/journal.pone.0044734

Singh, S. D., Robbins, N., Zaas, A. K., Schell, W. A., Perfect, J. R., and Cowen, L. E. (2009). Hsp90 governs echinocandin resistance in the pathogenic yeast Candida albicans via calcineurin. PLoS Pathog. 5:e1000532. doi: 10.1371/journal.ppat.1000532

Smith, D. A., Nicholls, S., Morgan, B. A., Brown, A. J., and Quinn, J. (2004). A conserved stress-activated protein kinase regulates a core stress response in the human pathogen Candida albicans. Mol. Biol. Cell 15, 4179-4190. doi: 10.1091/mbc.E04-03-0181

Smith, W. L., and Edlind, T. D. (2002). Histone deacetylase inhibitors enhance Candida albicans sensitivity to azoles and related antifungals: correlation with reduction in CDR and ERG upregulation. Antimicrob. Agents Chemother. 46, 3532-3539. doi: 10.1128/AAC.46.11.3532-3539.2002

Soo, E. T., Yip, G. W., Lwin, Z. M., Kumar, S. D., and Bay, B. H. (2008). Heat shock proteins as novel therapeutic targets in cancer. In Vivo 22, 311-315.

Soroka, J., Wandinger, S. K., Mäusbacher, N., Schreiber, T., Richter, K., Daub, H., et al. (2012). Conformational switching of the molecular chaperone Hsp90 via regulated phosphorylation. Mol. Cell 45, 517-528. doi: 10.1016/j.molcel.2011.12.031

Steinbach, W. J., Reedy, J. L., Cramer, R. A. Jr., Perfect, J. R., and Heitman, J. (2007). Harnessing calcineurin as a novel anti-infective agent against invasive fungal infections. Nat. Rev. Microbiol. 5, 418-430. doi: 10.1038/nrmicro1680

Sun, J. N., Solis, N. V., Phan, Q. T., Bajwa, J. S., Kashleva, H., Thompson, A., et al. (2010). Host cell invasion and virulence mediated by Candida albicans Ssa1. PLoS Pathog. 6:e1001181. doi: 10.1371/journal.ppat.1001181

Sun, S., Li, Y., Guo, Q., Shi, C., Yu, J., and Ma, L. (2008). In vitro interactions between tacrolimus and azoles against Candida albicans determined by different methods. Antimicrob. Agents Chemother. 52, 409-417. doi: 10.1128/AAC.01070-07

Sussman, A., Huss, K., Chio, L. C., Heidler, S., Shaw, M., Ma, D., et al. (2004). Discovery of cercosporamide, a known antifungal natural product, as a selective Pkc1 kinase inhibitor through high-throughput screening. Eukaryot. Cell 3, 932-943. doi: 10.1128/EC.3.4.932-943.2004
Tissières, A., Mitchell, H. K., and Tracy, U. M. (1974). Protein synthesis in salivary glands of Drosophila melanogaster: relation to chromosome puffs. J. Mol. Biol. 84, 389-398. doi: 10.1016/0022-2836(74)90447-1

Trojer, P., Brandtner, E. M., Brosch, G., Loidl, P., Galehr, J., Linzmaier, R., et al. (2003). Histone deacetylases in fungi: novel members, new facts. Nucleic Acids Res. 31, 3971-3981. doi: 10.1093/nar/gkg473

Umeyama, T., Kaneko, A., Niimi, M., and Uehara, Y. (2006). Repression of CDC28 reduces the expression of the morphology-related transcription factors, Efglp, Nrg1p, Rbf1p, Rim101p, Fkh2p and Tec1p and induces cell elongation in Candida albicans. Yeast 23, 537-552. doi: 10.1002/yea.1373

Uppuluri, P., Nett, J., Heitman, J., and Andes, D. (2008). Synergistic effect of calcineurin inhibitors and fluconazole against Candida albicans biofilms. Antimicrob. Agents Chemother. 52, 1127-1132. doi: 10.1128/AAC.01 397-07

Veri, A., and Cowen, L. E. (2014). Progress and prospects for targeting Hsp90 to treat fungal infections. Parasitology 141, 1127-1137. doi: $10.1017 /$ S0031182013002072

Vylkova, S., Jang, W. S., Li, W., Nayyar, N., and Edgerton, M. (2007). Histatin 5 initiates osmotic stress response in Candida albicans via activation of the Hog1 mitogen-activated protein kinase pathway. Eukaryot. Cell 6, 1876-1888. doi: 10.1128/EC.00039-07

Vylkova, S., Li, X. S., Berner, J. C., and Edgerton, M. (2006). Distinct antifungal mechanisms: $\beta$-defensins require Candida albicans Ssal protein, while Trk1p mediates activity of cysteine-free cationic peptides. Antimicrob. Agents Chemother. 50, 324-331. doi: 10.1128/AAC.50.1.324-331.2006

Wirk, B. (2011). Heat shock protein inhibitors for the treatment of fungal infections. Recent Pat. Antiinfect. Drug Discov. 6, 38-44. doi: 10.2174/157489111794407840

Xie, J. L., O'meara, T. R., Polvi, E. J., Robbins, N., and Cowen, L. E. (2017). Staurosporine induces filamentation in the human fungal pathogen Candida albicans via signaling through Cyr1 and protein Kinase A. mSphere 2:e0056-17. doi: 10.1128/mSphere.00056-17

Yu, Q., Ding, X., Zhang, B., Xu, N., Jia, C., Mao, J., et al. (2014a). Inhibitory effect of verapamil on Candida albicans hyphal development, adhesion and gastrointestinal colonization. FEMS Yeast Res. 14, 633-641. doi: 10.1111/1567-1364.12150

Yu, Q., Xiao, C., Zhang, K., Jia, C., Ding, X., Zhang, B., et al. (2014b). The calcium channel blocker verapamil inhibits oxidative stress response in Candida albicans. Mycopathologia 177, 167-177. doi: 10.1007/s11046-014-9735-7

Conflict of Interest Statement: The authors declare that the research was conducted in the absence of any commercial or financial relationships that could be construed as a potential conflict of interest.

Copyright (c) 2017 Gong, Li, Yu and Sun. This is an open-access article distributed under the terms of the Creative Commons Attribution License (CC BY). The use, distribution or reproduction in other forums is permitted, provided the original author(s) or licensor are credited and that the original publication in this journal is cited, in accordance with accepted academic practice. No use, distribution or reproduction is permitted which does not comply with these terms. 